Karlsruhe Institute of Technology

\title{
A complete EM analysis of DEMO WCLL Breeding Blanket segments during VDE-up
}

\author{
$\underline{\text { Ivan A. Maione }}^{\mathrm{a}}$, Massimo Roccellab ${ }^{\mathrm{b}}$, Flavio Lucca ${ }^{\mathrm{b}}$, Anna Marin $^{\mathrm{b}}$, Claudio Bertolinib
}

aKarlsruhe Institute of Technology (KIT), Hermann-von-Helmholtz-Platz 1, 76344 Eggenstein-Leopoldshafen, Germany

bLT Calcoli Srl, via Bergamo 60, 23807 Merate (LC), Italy

With regard to the Breeding Blanket (BB) project, a global DEMO EM model, suitable to account for different blankets designs, has been developed in previous years with the capability to analyse EM transients in presence of both toroidal and poloidal magnetic fields and considering materials with nonlinear magnetic properties. Using the FE model based on the WCLL design, a VDE-up with a $74 \mathrm{~ms}$ current quench time was analysed. The present work reports a complete EM analysis of the BB segments that takes into account eddy currents, the interaction of the BB magnetized material with the magnetic field (ferromagnetic forces) and the contribution of halo currents. Moreover, in order to follow the ongoing development of BB concepts, a comparison between the Single Module Segmentation (SMS) and Multi Module Segmentation (MMS) is also made, giving thus a complete view of the EM loads behaviour during the considered off-normal event.

Electromagnetic FEM model

20-degree sector model based on DEMO baseline 2015
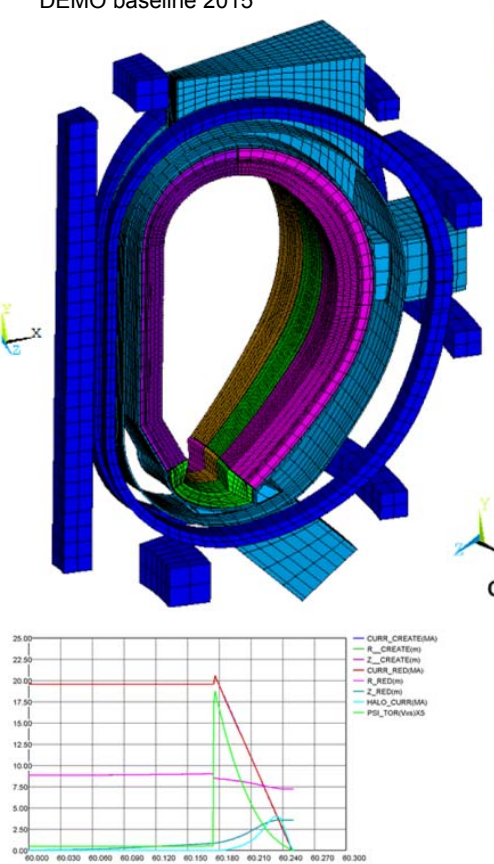

Poloidal coils' currents and plasma input (both poloidal and toroidal contribution) have been calculated by CarMa0NL code and provided for a VDE-up with a 74 ms current quench time.

Ferromagnetic forces

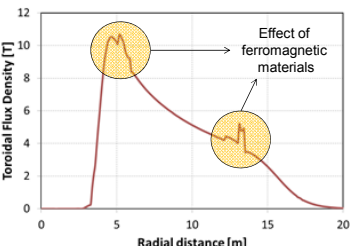

The generated magnetic field
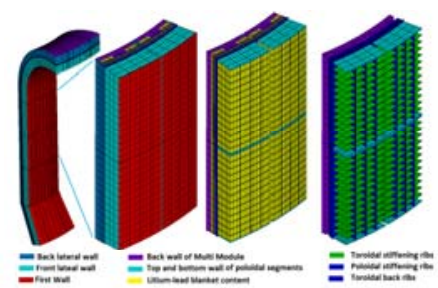

Averaged materials and EM properties have been used for the conductive components defined in the WCLL FEM model.

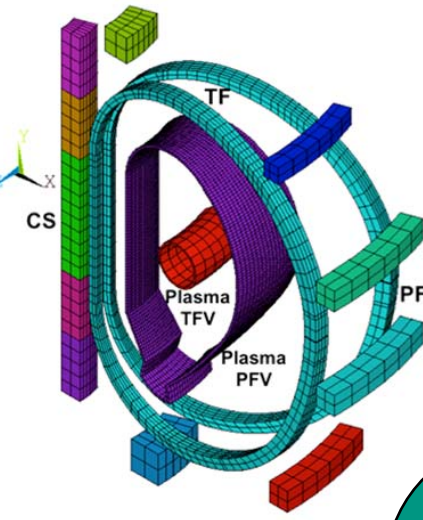

magnetises the structural components made in EUROFER97 producing forces that act during all the operational states of the machine.
- Lorentz forces
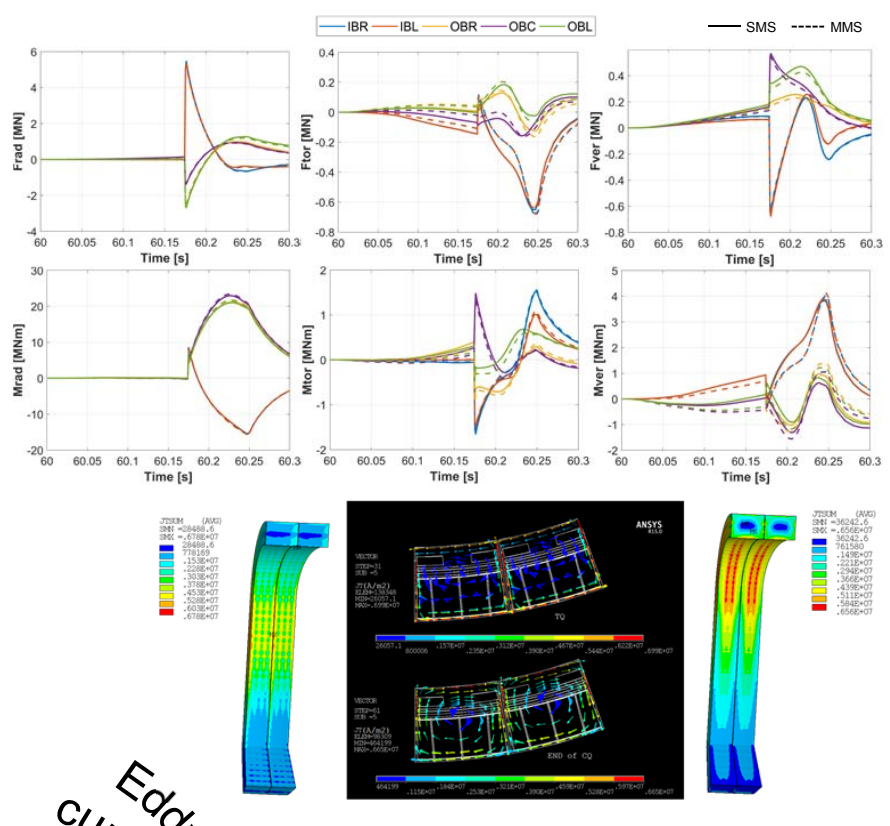

Two types of currents contribute to Lorentz forces:

$>$ eddy currents, caused by the decay of the plasma current and the change of plasma shape;

$>$ poloidal halo currents ( $\mathrm{HCs}$ ), which flow through the structure wetted by the disrupting plasma when it gets in contact with the surrounding structures.
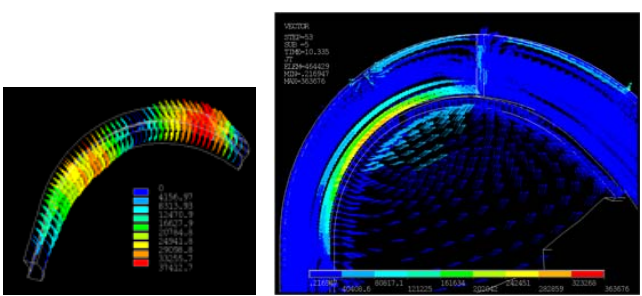
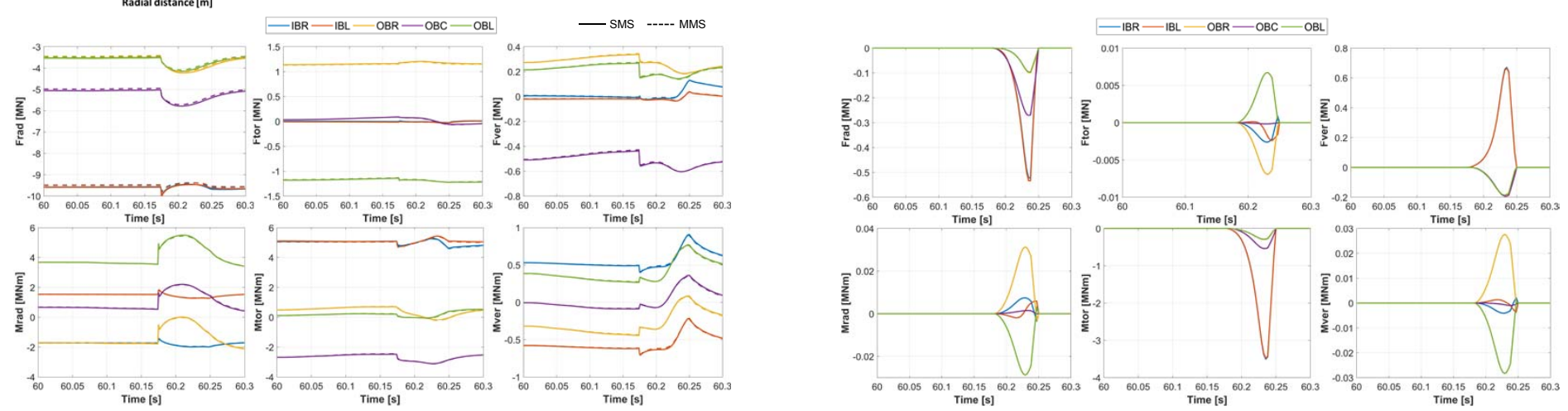